\title{
COVID-19 hinterlässt Spuren in der praxisambulanten Versorgung
}

\section{Urs Stoffel}

Dr. med., Mitglied des FMH-Zentralvorstandes, Departementsverantwortlicher Ambulante Versorgung und Tarife

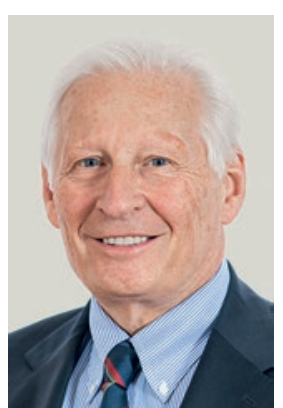

Die COVID-19-Pandemie hatte und hat erhebliche Auswirkungen auf die praxisambulante Versorgung in der Schweiz. Das vom Bundesrat angeordnete Verbot «nicht dringend angezeigte medizinische Behandlungen» während der Dauer des sechswöchigen «Lockdowns» zu unterlassen, führte bei den Arztpraxen und ambulanten ärztlichen Zentren in diesem Zeitraum zu Verlusten von 117 Mio. CHF. Die Kurzarbeitsentschädigung für Angestellte ist in dieser Zahl bereits berücksichtigt.

Fast alle Leistungserbringer wurden durch das Verbot zu massiven Vorhalteleistungen und damit zu ineffizienter Leistungserbringung gezwungen. Die Erwerbsausfallentschädigung (EO) für Praxisinhaber wurde de facto verwehrt bzw. nur für ausgesprochene Härtefälle gewährt.

Die praxisambulante Versorgung ist systemrelevant

Während sich die Spitäler auf eine drohende Welle von Patientinnen und Patienten mit schweren Krankheitsverläufen vorbereiten mussten und dies auch hervorragend getan haben, wurde in dieser Zeit die Grundversorgung der Bevölkerung durch die praxisambulanten Institutionen unter deutlich erschwerten Umständen gewährleistet und sichergestellt.

Die verschärften Hygienemassnahmen und Abstandsregeln führen im KMU Arztpraxis auch nach Aufhebung des Lockdowns zu anhaltenden Effizienzverlusten. Statistisch erreichten die Arztpraxen nach Ende des Lockdowns von Mai bis Juli lediglich 93\% der früher üblichen Leistungserbringung. Die Mindereinnahmen in diesem Zeitraum gegenüber dem Vorjahr betrugen weitere 147 Mio. CHF. Auch die vom BAG publizierten Zahlen des Monitorings der Krankenversicherungs-Kostenentwicklung (MOKKE) bestätigen diese Umsatzverluste von rund $5 \%$.

In diesem Jahr werden die erwähnten Umsatzeinbussen zu Einkommensverlusten von 20\% in den Arztpraxen und ambulante Zentren führen. Da die betriebswirtschaftlich übliche Reservebildung bei der
Erbringung von OKP-Leistungen ausgeschlossen ist (Art 59c, Abs. b, KVV), werden die niedergelassenen Ärztinnen und Ärzte mit einer Senkung ihrer Kosten reagieren müssen.

Die COVID-19-Pandemie verursacht aber nicht nur Einkommensverluste in der praxisambulanten Versorgung, sie führt auch zu erheblichen Mehrkosten. Seit Beginn des "Lockdowns» sind Behandlungen von Patientinnen und Patienten nur unter Anwendung von besonderen Schutzmassnahmen gegen das Coronavirus zugelassen. Ebenso muss auch das Praxispersonal mit Schutzmassnahmen geschützt werden. Dazu hat die FMH am 22. April 2020 ein Schutzkonzept vorgelegt, das festhält, wie Patientinnen und Patienten, Ärztinnen und Ärzte sowie das Praxispersonal vor einer Ansteckung mit dem Coronavirus geschützt werden müssen. Die Mehrkosten, bedingt durch zusätzliches Verbrauchsmaterial, verlängerte Wechselzeiten, Mehrkosten für Triage, Beratungen und Instruktionen am Telefon durch den Arzt oder die MPA und vermehrte Arbeitsausfälle, werden hochgerechnet für dieses Jahr weitere rund 445 Mio. CHF ausmachen.

Aufgrund unserer Auswertungen waren - auch auf dem Höhepunkt des Lockdowns - mehr als 60\% der Behandlungen in den Arztpraxen und ambulanten ärztlichen Zentren dringlich und wurden entsprechend durchgeführt. Weniger als $40 \%$, mussten - weil nicht dringlich - verschoben werden.

\section{COVID-19 verursacht nicht nur Einkommens-} verluste in der praxisambulanten Versorgung - sie führt auch zu erheblichen Mehrkosten.

Wegen der Verluste infolge COVID-19-induzierter Mehrkosten (Vorhalteleistungen und Effizienzverluste), drängt sich eine temporäre Tarifanpassung (z.B. über den Taxpunktwert) auf.

Zur Finanzierung dieser Tarifanpassung stehen einerseits der Bund, der nicht dringend angezeigte medizinische Behandlungen temporär verboten hat, und andererseits die Krankenversicherer, die für unveränderte Prämien weniger Kosten finanzieren müssen, gemeinsam in der Pflicht. 